\title{
Vascular sclerosing effects of bleomycin on cutaneous veins: a pharmacopathologic study on experimental animals ${ }^{*}$
}

\author{
Khalid M AlGhamdi ${ }^{1,2}$ \\ Abdelkader E. Ashour ${ }^{3}$ \\ Abdullah Hasan AlOmrani ${ }^{5}$
}

\author{
Ashok Kumar ${ }^{2}$ \\ Ammar C. AL-Rikabi ${ }^{4}$ \\ Shaik Shaffi Ahamed ${ }^{6}$
}

DOI: http:/ / dx.doi.org/10.1590/abd1806-4841.20175851

\begin{abstract}
BACKGROUND: Varicose veins and the complications of venous disease are common disorders in humans.
ОвјестіуЕ: To study the effects of bleomycin as a potential new sclerosing agent and its adverse events in treating varicose veins. MEtHods: Bleomycin-loaded liposomes $0.1 \mathrm{ml}$ was injected in the dorsal ear veins of white New Zealand rabbits. Sodium tetradecyl sulfate was used as a positive control. Normal saline was used as negative control. The blood vessels of the treated ears were photographed before and at one hour and two, eight and 45 days after treatment. Biopsies from the treated areas were obtained for histological examination. Blood samples were collected to determine any possible toxicity.

REsults: Bleomycin by itself was ineffective; therefore, liposomes were used as a vector to deliver bleomycin to the vein lumen. Subsequently, bleomycin started showing its sclerosing effects. Toxicity monitoring showed no apparent hematologic, pulmonary, hepatic or renal toxicities. This study revealed that bleomycin induced vasculitis, which led to vascular occlusion, which was observed on day 1 and day 8 . No bleomycin-related injury was noted by histopathological examination of lung sections. The calculation of the lung/body weight coefficient indicated that edema was present in the experimental groups compared with the negative and positive controls.

STUDY LIMITATIONS: Relatively small number of experimental animals used.

Conclusions: This study showed that bleomycin-loaded liposomes were able to induce vasculitis and vascular occlusion without any toxicity or complications. It might be useful, hence, to treat patients suffering from Varicose veins and other ectatic vascular diseases with this agent.
\end{abstract}

Keywords: Bleomycin; Rabbits; Sclerosing solutions; Varicose veins; Vasculitis

\section{INTRODUCTION}

Varicose veins (VV) and the complications of venous disease represent an exceedingly common group of disorders. It is estimated that up to $73 \%$ of women and $56 \%$ of men have VVs. ${ }^{1}$ They have a deleterious effect on disease-specific and generic health-related quality of life. In addition, the management of these conditions is a major source of health service expense. Affected people can develop complications in the form of superficial thrombophlebitis, disorders of pigmentation, lipodermatosclerosis, hemorrhage, ulceration, stasis dermatitis and increased risk of deep vein thrombosis. Many affected people are in their most productive professional years. Millions of workdays each year are lost as a result of absence because of venous disease. Because the etiology is elusive, there is no specific means of prevention, and the problem has been increasing. ${ }^{1-3}$ Sur- gery and sclerotherapy have been the two main approaches to treating VVs for many years. However, surgery has many complications, such as prolonged recovery periods and significant recurrence rates. In addition, all of the currently available sclerosing agents cause various adverse events and have significant recurrence rates. ${ }^{4}$ Thus, new approaches for VV therapy are urgently needed.

Bleomycin, a widely used antitumor antibiotic, has been anecdotally reported to have some antiangiogenic effects. ${ }^{5,6}$ On the basis of these findings, we utilized bleomycin as a sclerosing agent to treat VVs in an experimental animal model. To cause sclerosant effects, bleomycin must penetrate the endothelial cells of the vein wall (tunica intima). However, its hydrophilicity (the estimated log $P$ is -9.7) and large molecular weight (1415 Da) hinder the penetra-

\footnotetext{
Received on 30.03.2016.

Approved by the Advisory Board and accepted for publication on 02.05.2016.

* Study conducted at the College of Medicine, King Saud University - Riyadh, Saudi Arabia.

Financial support: King Abdulaziz City for Science and Technology (KACST), Riyadh, Kingdom of Saudi Arabia (Project No. LGP-15-31).

Conflict of interest: None.

Dermatology Department, College of Medicine, King Saud University - Riyadh, Saudi Arabia.

Vitiligo Research Chair, College of Medicine, King Saud University - Riyadh, Saudi Arabia.

Department of Pharmacology and Toxicology, College of Pharmacy, King Saud University - Riyadh, Saudi Arabia.

Pathology Department, College of Medicine, King Saud University - Riyadh, Saudi Arabia.

Nanomedicine unit (NMU-KSU), Pharmaceutical Department, College of Pharmacy, King Saud University - Riyadh, Saudi Arabia.

Department of Family \& Community Medicine, College of Medicine, King Saud University - Riyadh, Saudi Arabia. 
tion of bleomycin through the endothelial cells. It has been shown that bleomycin is a drug with relatively low permeability and minimal intracellular concentrations. ${ }^{7,8}$ Therefore, the rate-limiting step of the sclerosant effect of bleomycin lies in its membrane permeability. Alternative strategies have been investigated to enhance the permeability of drugs with low permeability through biological membranes. Among these strategies, liposomes have been observed to constitute the safest and most promising approach due to their biodegradability and biocompatibility. The aim of this study was to investigate the effects of bleomycin-loaded liposomes on the treatment of VVs using sodium tetradecyl sulfate (STS), which is the strongest FDA-approved sclerosant, ${ }^{9,10}$ as a potential control.

\section{METHODS}

Sclerosing agents were injected into rabbits' dorsal ear veins after clamping both sides of the vein. Morphologic and histopathological changes were observed, recorded and analyzed. The rabbits' dorsal ear vein was chosen because it is clearly visible and easy to inject and handle.

\section{Preparation of Liposomes}

Liposomes were prepared by the dehydration-rehydration method as described by Kirby and Gregoriadis, ${ }^{11}$ with some modifications. Briefly, phospholipids and cholesterol were mixed at the molar ratios shown in table 1 with $50 \mu \mathrm{l}$ of ethanol, and they were maintained at $60^{\circ} \mathrm{C}$ until a clear solution was formed. Liposomes were formed when $10 \mathrm{ml}$ of bleomycin solution $(2 \mathrm{mg} / \mathrm{ml})$ containing sorbitol $(10 \mathrm{mg} / \mathrm{ml})$ was added to the phospholipid/cholesterol solution. The liposome dispersion was kept at $4^{\circ} \mathrm{C}$ overnight to obtain complete swelling. A probe sonicator was used for two minutes to reduce vesicle size before lyophilization. The lyophilized liposomes were then rehydrated with $10 \mathrm{ml}$ of water before conducting the experiment.

\section{Laboratory Animals}

The study included 18 13-week-old New Zealand rabbits, weighing approximately $2.0 \mathrm{~kg}$ each. The dorsal lateral parts of the rabbits' left ears were shaved off. All of the animals were treated in a humane manner. The study was approved by the institutional research board at College of Medicine, King Saud University, Riyadh, Saudi Arabia.

\section{Experimental Groups}

The study included a total of three groups (six rabbits each), namely negative control, positive control and experimental groups. The negative control group animals received sterile saline solution, whereas in the positive control group, $1 \%$ STS was injected. ${ }^{12}$ The experimental group rabbits received bleomycin solution $(2 \mathrm{mg} / \mathrm{ml})$.

\section{Methodology of Injections}

The dorsal left ear veins of non-anesthetized rabbits were injected with $0.1 \mathrm{ml}$ of the positive and negative sclerosing agents, as well as bleomycin. ${ }^{13}$ After injection, ten-minute distal occlusion of the dorsal vein of the rabbit's left ear was achieved using a surgical clamp to prevent the injected drugs from reaching the systemic circulation.

\section{Data Collection and Assessment}

The treated rabbit's dorsal ear blood vessels were photographed before treatment as a baseline. They were also photo- graphed at 1 hour and 1,8, and 45 days after treatment. Thereafter, three rabbits from each group were anesthetized with ketamine injections to obtain biopsies from the treated areas for histological examinations. The biopsies were performed under aseptic conditions with a 4-mm punch biopsy dermatologic instrument. The samples were then kept in $10 \%$ formalin saline solution until the time of histopathological processing.

\section{Histologic Assessment}

The tissue samples obtained were processed, paraffin embedded and sectioned according to standard methods using Tissue-Tek TEC and Tissue-VIP (Sakura Finetek USA, Inc. Torrance, CA, USA) using an embedding agent and tissue processor, respectively. Several $5-\mu \mathrm{m}$-thick sections were subsequently stained with hematoxylin and eosin using a Leica ST 520 automated stainer (Wetzlar, Germany). Presence of inflammation, vascular proliferation, lumen narrowing and fibrosis was assessed and recorded. Diameters of the blood vessels were assessed microscopically (Nikon - eclipse 80i, Melville, NY, USA) using the standard ruler on the microscope stage. Additional morphologic features were also described, including hemorrhage, edema, eosinophilic infiltration, granulomatous reaction, necrosis and ulceration.

\section{Toxicity Monitoring}

Blood was collected from the marginal ear vein five days before treatment (as a baseline), and further blood samples were collected at 1 hour and 1, 8 and 45 days post-treatment. Blood was kept for 30 minutes at room temperature, followed by 2 hours on ice to induce clotting and centrifugation at 3,000 $\mathrm{g}$ for 10 minutes to separate the serum, which was then stored at $-80^{\circ} \mathrm{C}$ until analysis. Toxicity of bleomycin was monitored by conducting a complete blood count and liver and kidney function tests on randomly selected subgroups $(n=3)$ of treated rabbits.

\section{Hematologic Monitoring}

A complete blood count was performed on an aliquot of the blood sample using an automated analyzer (Sysmex XE-2100, Sysmex Corporation, Kobe, Japan).

\section{Assessment of Liver Function}

Markers of hepatocyte necrosis and aspartate aminotransferase (AST) and alanine aminotransferase (ALT) activities in serum were determined ${ }^{14}$ using commercially available diagnostic kits (Biosystems, Barcelona, Spain).

\section{Assessment of Kidney Function}

Blood urea nitrogen (BUN) levels were measured in serum with the colorimetric, end point BUN diagnostic kit (Sigma, St. Louis, MO, USA). Serum creatinine levels were also determined with the colorimetric end point creatinine diagnostic kit (Sigma, St. Louis, MO, USA).

TABLE 1: Molar ratios of phospholipids and cholesterol

\begin{tabular}{cccc} 
& PL 90 & CH & Total \\
\hline $\begin{array}{c}\text { Molar ratio } \\
\begin{array}{c}\text { Weight for } \\
\text { 100mg/10mL } \\
\text { formula }\end{array}\end{array}$ & 10 & 5 & 15 \\
& 79.7 & 20.3 & 100 \\
\end{tabular}

PL 90: Soybean lecithin Lipoid S100; CH: cholesterol 


\section{Evaluation of Pulmonary Toxicity}

The following tests were performed on subgroups $(n=3)$ of rabbits to assess the extent of bleomycin-induced pulmonary toxicity.

\section{Lung Histologic Analysis}

Toxicity of the drug was monitored by conducting histological examinations of lung tissue. Lung damage was assessed histopathologically after euthanasia by inflating and fixing the left lung in $10 \%$ formalin-saline solution, embedding the selected tissue in paraffin and processing the fixed tissue to obtain sections for microscope slides. ${ }^{15}$ These 5 - $\mu \mathrm{m}$ sections were then stained with hematoxylin and eosin. Slides were evaluated histopathologically under a light microscope by an experienced pathologist.

\section{Assessment of Lung edema}

Degree of lung edema was evaluated by calculating the lung weight coefficient (LWC) factor using the following formula: LWC $=$ (lung weight $/$ body weight $) \times 100 \%$. Rabbits were weighed before being sacrificed (body weight). Lungs were weighed after being removed from the trachea (lung weight). The lung/body weight coefficient was calculated as previously reported. ${ }^{16}$

\section{Statistical Analysis}

The data were analyzed using Statistical Package for the Social Sciences statistical software, version 21.0, SPSS Inc., Chicago, IL, USA. Tables were prepared to visualize the variations in blood test results among the experimental groups, as well as the variation within each group over time. Descriptive statistics (mean and standard deviation) were used to describe the quantitative outcome variables. One-way analysis of variance was used to compare the mean values of quantitative variables across the categorical variables, followed by Duncan's multiple range test, to determine whether there were any differences between the groups and over time within each group. A p-value of $\leq 0.05$ was considered statistically significant.

\section{RESULTS}

Bleomycin did not show any therapeutic effects on animals when it was injected alone (data not shown). Therefore, liposomes were used as a vector to deliver bleomycin into the vein. The visible effects and histopathological changes were observed, recorded and analyzed. In addition, the toxicity, as well as the recurrence rate, was monitored and compared.

\section{Bleomycin-induced Vascular Occlusion and Vasculitis}

Histological findings indicate that 2 of 6 tested animals showed evidence of vascular occlusion after 1 hour and at 45 days from bleomycin-loaded liposome injection. The luminal obstruction was estimated to be 50\% (Table 2 and Figures 1 and 2). Eosinophilic venulitis was noted, however, at days 1 and 8 after injection. STS caused lumen narrowing in animals in the positive control group. Normal saline, the negative control, showed no effects as expected. Results of the histologic assessment of the injected blood vessels are summarized in table 2.

\section{Lung, Heart and Kidney Pathology}

No bleomycin-induced injury was noted upon examination of the lung, liver or heart sections obtained from experimental animals. Eosinophilic bronchopneumonia and focal interstitial pneumonitis, most likely caused by an acquired infection in the animal hous- ing, were, however, observed in one positive control group animal.

\section{Assessment of Lung edema}

The calculation of the lung/body weight coefficient indicated that edema was present in the experimental groups compared with the negative and positive control groups (Table 3).

\section{Toxicity Monitoring}

None of the hematologic or biochemical laboratory measurements showed any differences among the studied groups. There were no statistically significant differences in the mean values of AST, ALT, creatinine, BUN, hemoglobin, RBC, WBC or platelet count across the 3 groups (Table 4 ).

\section{DISCUSSION}

One of the most important concepts in sclerotherapy is achieving optimal destruction of the blood vessel wall with the minimum concentration of sclerosant because high concentrations can lead to adverse reactions. ${ }^{17}$ The purpose of this study was to determine whether bleomycin, as a new sclerosant, could produce significant sclerosing effects after injecting a minimal dose that would yield optimal results and reduce the incidence and severity of adverse sequelae.

VVs are twisted, enlarged veins near the surface of the skin that appear dark blue. They most commonly develop in the legs and ankles and cause heaviness, tiredness, aching discomfort and pain for patients. ${ }^{18}$ VVs develop when valves become faulty and vein walls become weakened. Normally, the one-way valves in healthy veins keep blood flowing efficiently against gravity toward the heart. When these valves do not function properly, blood pools, pressure increases, and the veins become weakened, enlarged and twisted. This result is called venous insufficiency. ${ }^{19}$ Symptoms can be worse after standing or sitting for long periods of time. VVs can also cause skin color changes (stasis pigmentation), dry and thinned skin, skin inflammation, open sores (ulcerations) or bleeding after minor injuries. Some people may be more likely than others to develop VVs because of inherited characteristics (genetic etiology), obesity, pregnancy, intra-abdominal malignancy and aging process. ${ }^{20}$

Despite these problems, most patients do not undergo any treatment for long periods of time, even when complications arise, mainly because the only definitive therapeutic option is surgery, which is not only invasive but is also associated with surgical complications, such as increased in-hospital costs, prolonged recovery periods and significant recurrence rates. ${ }^{21-23}$ Despite improvements in preoperative evaluations and methods of treatments, recurrence following VV surgery has been reported to occur in $20 \%$ and $80 \%$ of cases. ${ }^{24-25}$

Sclerotherapy has been advocated as a VV treatment for over 40 years. ${ }^{26}$ It has been suggested as an alternative to surgical treatment or to be used in combination with it. It can reduce costs, and it offers the possibility of treatment to more patients within cash-limited health services. ${ }^{27}$ It involves the deposition of a foreign substance into a vessel lumen, resulting in thrombosis and fibrosis. Sclerosants initiate endothelial damage. Destruction of the endothelial layer with the resulting exposure of subendothelial collagen leads to platelet aggregation and activation of the intrinsic coagulation pathway. ${ }^{13}$ These events, in turn, prompt endofibrosis, which ultimately ablates the vein. 
There are numerous sclerosing agents used currently. However, no currently available sclerosant method has all of the attributes of the perfect sclerosing agent. All currently available sclerosants cause various adverse events and are associated with significant recurrence rates. The strongest and most common sclerosing agent currently available is STS. ${ }^{4}$ An anionic detergent, STS has been used for the treatment of VVs since 1946. Some of the most common ad- verse events are residual pigmentation and thrombophlebitis. Other adverse events include necrosis and ulceration, which are usually rare and caused by leakage of the sclerosing agent from the vein due to using too high concentrations. Allergic reactions are rare, and they present as local or generalized rash, urticaria, nausea or vomiting, asthma and vascular collapse. Anaphylactic shock, which may be fatal, is extremely rare but remains a possibility.

TABLE 2: Comparison of two types of scars

Findings at 1 hour after injection

\begin{tabular}{|c|c|c|c|c|c|c|}
\hline Formulations & Case no. & Inflam. & Fibrosis & $\begin{array}{l}\text { Vascular thrombosis } \\
\text { and/or obliteration }\end{array}$ & Eosinophils & Other features \\
\hline $\mathrm{NaCl}^{\mathrm{a}}$ & $204 \& 205$ & Mild & None & None & None & Mild edema \\
\hline \multirow{2}{*}{$1 \% \mathrm{STS}^{\mathrm{b}}$} & 209 & Mild & None & None & None & None \\
\hline & 210 & Mild & None & Present & None & Mild edema \\
\hline \multirow{2}{*}{$\mathrm{BL}^{*}$} & $230 \& 233$ & Moderate & None & None & None & None \\
\hline & 234 & Minimal & None & Present & None & Mild edema \\
\hline \multicolumn{7}{|c|}{ Findings at Day 1 after injection } \\
\hline \multirow{3}{*}{$\mathrm{NaCl}^{\mathrm{a}}$} & 203 & Mild & Mild & None & Present & Edema and focal necrosis \\
\hline & 204 & None & None & Present & None & Interstitial hemorrhage \\
\hline & 205 & Moderate & None & None & None & None \\
\hline \multirow{2}{*}{$1 \%$ STS $^{b}$} & $207 \& 210$ & Marked & None & None & Present & Edema \\
\hline & 208 & Mild & None & Present & Present & Edema and vasculitis \\
\hline \multirow{2}{*}{$\mathrm{BL}^{*}$} & $228 \& 231$ & Moderate & None & None & Present & Marked edema \\
\hline & 229 & Mild & None & None & None & None \\
\hline \multicolumn{7}{|c|}{ Findings at Day 8 after injection } \\
\hline \multirow{3}{*}{$\mathrm{NaCl}^{\mathrm{a}}$} & 201 & Minimal & None & None & None & None \\
\hline & 202 & Mild & None & None & Mild & Interstitial hemorrhage \\
\hline & 204 & Mild & Present & None & Present & Edema $(2+)$ \\
\hline \multirow{3}{*}{$1 \% \mathrm{STSb}$} & 207 & Marked & Marked & None & Present & Ulcer \\
\hline & 208 & Extensive & Present & None & Present & Epithelial necrosis seen \\
\hline & 209 & Moderate & None & None & None & Artifacts seen in tissue \\
\hline \multirow{2}{*}{$\mathrm{BL}^{*}$} & $230 \& 232$ & Mild & Mild & None & None & None \\
\hline & 234 & Moderate & Absent & None & None & Interstitial hemorrhage \\
\hline \multicolumn{7}{|c|}{ Findings at Day 45 after injection } \\
\hline \multirow{2}{*}{$\mathrm{NaCl}^{\mathrm{a}}$} & 201 & Marked & Mild & None & Numerous & Ulceration \\
\hline & $203 \& 204$ & Mild & Mild & None & None & Mild edema \\
\hline \multirow{3}{*}{$1 \% \mathrm{STS}^{\mathrm{b}}$} & 207 & Moderate & Present & None & Mild & Dystrophic calcifications \\
\hline & 208 & Moderate & Mild & Present & None & Necrotic cartilage \\
\hline & 210 & $\begin{array}{l}\text { Marked + } \\
\quad \text { ulcer }\end{array}$ & Moderate & None & Present & None \\
\hline \multirow{2}{*}{$\mathrm{BL}^{*}$} & 228 & Mild & Mild & None & None & None \\
\hline & 229 & None & Marked & Present & None & None \\
\hline
\end{tabular}

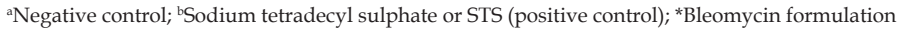


In this study, bleomycin was introduced as an alternative to STS and was used as sclerosing agent to treat VVs. The rationale for choosing bleomycin as a sclerosing agent was based on a case report, in which intralesional injections of bleomycin successfully palliated severe recurrent epistaxis for almost two years without the need for adjuvant therapy in a patient with hereditary hemorrhagic telangiectasia. ${ }^{6}$

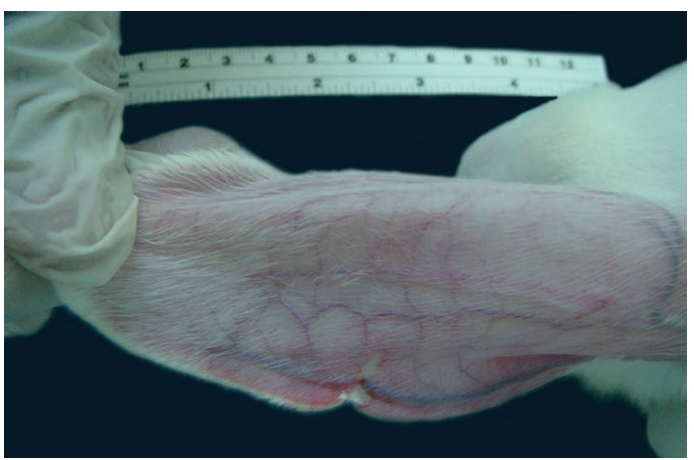

Figure 1: Rabbit ear 1 hour after bleomycin injection
In this study, we achieved $50 \%$ occlusion in the veins of bleomycin-loaded liposome-injected animals. Occlusion occurred because of the formation of organized thrombi associated with fibromuscular proliferation. Only thrombi that were adherent to the vascular wall were counted. It is important to mention, however, that an adherent and organizing thrombus was noted in one of the control animals (Figure 3). It is possible that this thrombus was simply induced by the trauma of the injecting needle. In addition, marked eosinophilic vasculitis, characterized by perivascular edema, fibrinoid necrosis and eosinophilic infiltration, was seen (Figure 3).

\begin{tabular}{cccc}
\multicolumn{3}{c}{ TABLE 3: Lung/body coefficients (mean \pm SD) } \\
$\begin{array}{c}\text { each group } \\
\text { Group }\end{array}$ & Lung/body weight coefficient (\%) & CH & Total \\
\hline Saline * & $0.56 \pm 0.02$ & 5 & 15 \\
STS ** & $0.49 \pm 0.09$ & 20.3 & 100 \\
BL $^{* * *}$ & $0.75 \pm 0.02$ & &
\end{tabular}

* Negative control; ** Sodium tetradecyl sulphate (positive control); ${ }^{* * * B l e o m y c i n ~ f o r m u l a-~}$ tion. Two animals per group were included to calculate the lung/body coefficient.
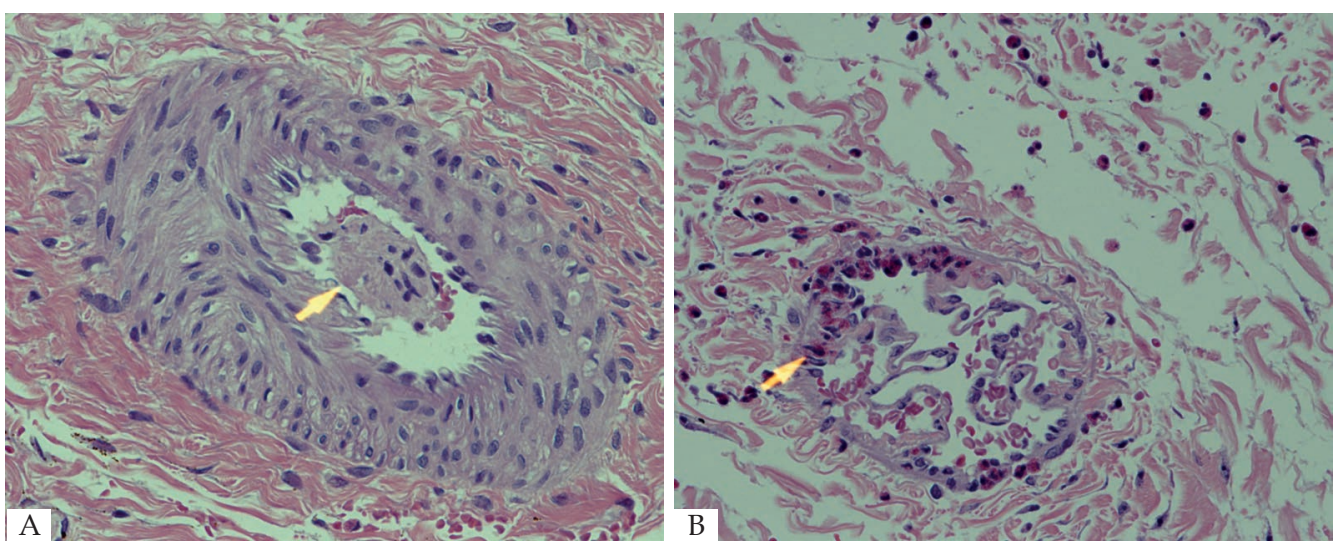

Figure 2: A)A small, organizing thrombus is seen adhering to the wall of an arteriole (arrowhead). The thrombus consisted of fibrin with spindle cell fibroblastic proliferation. The occlusion obtained in this case constituted approximately 50\% of the entire lumen surface. (Hematoxylin \& eosin stain, X 400). B) Eosinophilic venulitis in a medium sized venule was observed after 1 hour of injection. Note the presence of numerous eosinophils (arrow head) within the blood vessel wall. This is associated with endothelial cell detachment and perivascular edema. (Hematoxylin \& eosin stain, X400)
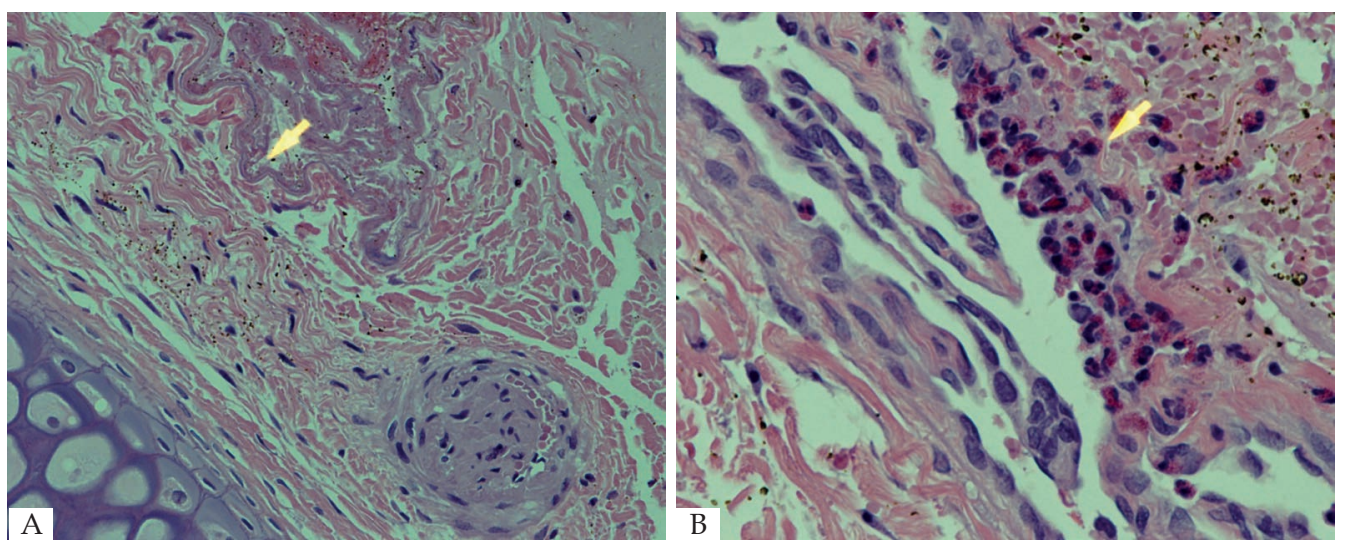

Figure 3: A) A completely necrotic venule (arrowhead) with an adjacent smaller vessel containing an adherent and organizing thrombus (right corner of the picture). Note the presence of significant fibrinoid necrosis and the almost complete loss of blood vessel detail in the affected necrotic venule. (Hematoxylin \& eosin stain, X400) B) Marked eosinophilic venulitis. Note the presence of numerous eosinophils (arrowhead) in the subendothelial layer within the blood vessel wall. (Hematoxylin \& eosin stain X600) 
It is also important to mention that eosinophilic venulitis was noticed at days 1 and 8 after injection. We believe that this event represents a form of bleomycin-induced vasculitis, which may be followed by fibrosis and vascular lumen obstruction. It is also well known that almost all vascular sclerosing agents can cause variable degrees of perivascular and vascular inflammation. This reaction, like all inflammatory reactions, will eventually lead to vascular obliteration with surrounding fibrosis, which is a desirable effect in cases of vascular ectasia and VVs. Alternative mechanisms have been proposed to explain the enhancement of drug permeation through biological membranes. It was proposed that liposomes might penetrate into the biological membrane by adhering onto the surface of the cell layer and subsequently fusing and interacting with, or destabilizing, the lipid matrix of the cell membrane. Liposomes may also enhance the penetration of molecules by loosening the lipid structure of the cell membrane and altering the barrier function of these cells against molecules, with a subsequent increase in the epithelial partitioning of the molecules. ${ }^{28}$

Bleomycin did not induce any injury to the lungs, livers or hearts of the treated animals. Bleomycin lung toxicity is known to be characterized by the presence of diffuse alveolar damage with hya-

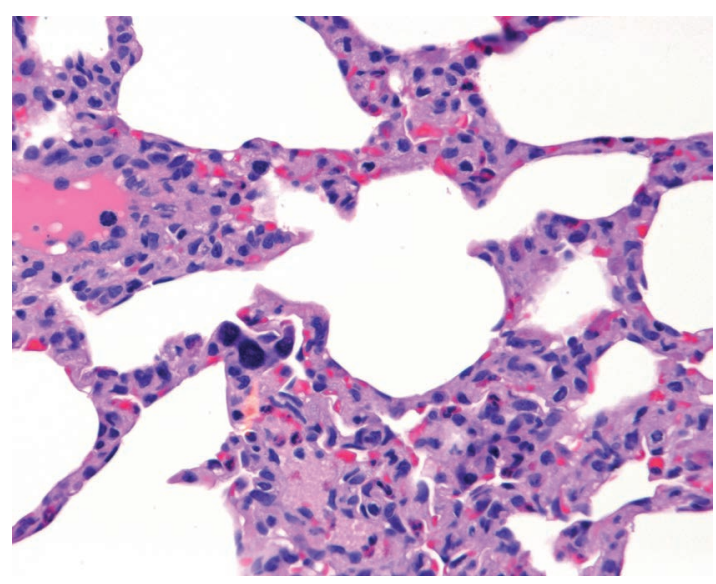

FigURE 4: Focal interstitial pneumonitis with excess eosinophils is seen. Scanty atypical type 2 pneumocytes were also observed in an animal from the control group, perhaps due to secondary infection acquired from the animal housing. (Hematoxylin \& eosin stain X400) line membrane formation, usually followed by type 2 pneumocyte proliferation and organizing pneumonia. Such changes were not observed during our experiments. However, a total of 7 animals from the negative and positive control groups showed variable degrees of eosinophilic bronchopneumonia and mild interstitial pneumonitis, most likely due to an acquired infection from the animal housing. Focal interstitial pneumonitis with excess eosinophils and scanty atypical type 2 pneumocytes were also observed in one positive control animal (Figure 4).

There were no statistically significant differences in the mean values of AST, ALT, creatinine, BUN, hemoglobin, RBC, and WBC across the three groups (Table 4). However, there were statistically significant differences in the mean platelet counts among the three groups $(F=3.35, p=0.045)$. By multiple comparison testing, it was observed that the mean platelet count was significantly higher in Group 3 (bleomycin), than the mean values in Group 1 (negative control) and Group 2 (positive control).

There were high and statistically significant differences in the mean values of AST, ALT, creatinine, BUN, hemoglobin, RBC, WBC and platelet count across the 5 time points (baseline, 1 Hour, Day 1, Day 8 and Day 45). By multiple comparison testing, it was observed that the mean values of AST were significantly higher on Day 8 and Day 45; ALT values were higher at 1 Hour and Day 8; creatinine values were higher on Day 45; hemoglobin values were higher at 1 Hour and Day 45; RBC values were higher at 1 hour; WBC values were higher at Day 45; and platelet count values were higher on Day 8 than at the other time points (Table 5).

Toxicity monitoring showed no apparent hematologic, pulmonary, hepatic or renal toxicities associated with the bleomycin used in this study. There were no significant differences among the various groups, indicating no difference in results among the positive control, negative control and bleomycin-treated animals. These results indicated that bleomycin was not more toxic than STS. The dose of bleomycin used in this study was extremely low. However because bleomycin has never been used to treat VVs, it was necessary to monitor toxicity.

One major apparent advantage of bleomycin sclerotherapy is the relatively minimal inflammatory reaction and edema post-injection. ${ }^{29}$ Bleomycin is rapidly absorbed after administration, and the peak concentration is obtained after approximately 45 minutes, with a half-life clearance of approximately 2.5 hours. High concen-

\begin{tabular}{|c|c|c|c|c|c|}
\hline Study variables & Gp 1 & Gp 2 & Gp 3 & F-value & p-value \\
\hline AST & $37.64(7.9)$ & $32.92(12.2)$ & 35.27 (11.6) & 0.66 & 0.524 \\
\hline ALT & 44.11(18.4) & 37.69 (11.6) & 49.333 (16.8) & 1.86 & 0.17 \\
\hline Creatinine & $0.62(0.1)$ & $0.71(0.2)$ & $0.7(0.1)$ & 1.41 & 0.255 \\
\hline BUN & $19.29(5.9)$ & $20.08(4.9)$ & $21.80(6.6)$ & 0.69 & 0.508 \\
\hline Hemoglobin & $12.04(1.1)$ & $11.37(0.9)$ & $11.89(1.2)$ & 1.47 & 0.243 \\
\hline RBC & $5.82(0.6)$ & $5.72(0.6)$ & $5.75(0.7)$ & 0.08 & 0.919 \\
\hline WBC & $8226.43(4022.3)$ & 7581.54 (2589.8) & 8805.33 (2913.1) & 0.49 & 0.612 \\
\hline Platelet count & 462785.71 (150547.3) & $571230.77(142150.3)$ & $618800(194356.5)$ & 3.35 & 0.045 \\
\hline
\end{tabular}

Gp 1: Negative control; Gp 2: Sodium tetradecyl sulphate (positive control);Gp 3: Bleomycin formulation. 


\begin{tabular}{cccccccc}
\multicolumn{6}{c}{ TABLE 5: Comparison of mean values of biochemical and hematological study variables across the five time points } \\
Study variables & BL & $\mathbf{1}$ hour & Day 1 & Day 8 & Day 45 & F-value & p-value \\
\hline AST & $32.63(9.8)$ & $31.71(7.7)$ & $29.67(7.5)$ & $37.11(11.4)$ & $44.44(10.5)$ & 3.3 & 0.021 \\
ALT & $28.13(13.9)$ & $52.92(16.9)$ & $43.78(11.9)$ & $51(15.2)$ & $44.33(15)$ & 3.56 & 0.015 \\
Creatinine & $0.63(0.2)$ & $0.62(0.1)$ & $0.62(0.1)$ & $0.67(0.1)$ & $0.84(0.1)$ & 4.62 & 0.004 \\
BUN & $16.38(3.1)$ & $22.14(5.6)$ & $17.33(6.2)$ & $24.56(6.9)$ & $21.67(2.9)$ & 3.73 & 0.012 \\
Hemoglobin & $11.59(0.9)$ & $12.87(0.7)$ & $10.9(0.9)$ & $11.64(0.9)$ & $12.11(1.1)$ & 5.1 & 0.002 \\
RBC & $5.53(0.4)$ & $6.46(0.3)$ & $5.29(0.3)$ & $5.82(0.7)$ & $5.85(0.7)$ & 5.26 & 0.002 \\
WBC & $6687.50(4169)$ & $8945.71(1858.3)$ & $6786.67(2282)$ & 8114.44 & $10620(2163.7)$ & 2.63 & 0.05 \\
& & & & $(3634.1)$ & & & \\
Platelet count & $(146402.5)$ & $(93087.5)$ & $(122979.4)$ & $(252434)$ & $(131180.2)$ & 2.66 & 0.048
\end{tabular}

trations of bleomycin can be toxic. Acute toxicity occurs when the dose exceeds 150 U. ${ }^{30}$ However, the bleomycin dose used in this study was very small. The maximum dose used in our study was $0.2 \mathrm{U}$ per animal. In addition, after the injection, ten-minute distal occlusion of the dorsal vein of the rabbits' left ears was achieved to prevent the injected drugs from reaching the systemic circulation. Therefore, we anticipate that bleomycin, at this low dose level and due to the occlusion applied, would be safe.

\section{CONCLUSIONS}

This study introduced the use of a new sclerosing agent for the treatment of VVs, which currently have no permanent cure without complications or side effects. Bleomycin-induced vasculitis was observed on Day 1 and Day 8 when bleomycin-loaded liposomes were used. In addition, organized thrombi with at least $50 \%$ luminal obstruction were seen at 1 hour and 45 days in two animals. The strength of this study lay in its proposal of a new sclerosing agent with the lowest possible concentration, yielding optimal results with minimal adverse events. The limitation of this pilot study was the relatively small number of experimental animals used. We strongly feel that the bleomycin dose used is the lowest possible; therefore, it is likely to be safe for use in further experiments. Additional larger studies are needed, however, before we can recommend bleomycin-loaded liposomes as a potential treatment for human VVs.」

\section{ACKNOWLEDGMENTS}

The authors are grateful to the King Abdulaziz City for Science and Technology (KACST), Riyadh, Kingdom of Saudi Arabia, for funding this project (No. LGP-15-31).

\section{REFERENCES}

1. Beebe-Dimmer JL, Pfeifer JR, Engle JS, Schottenfeld D. The epidemiology of chronic venous insufficiency and varicose veins. Ann Epidemiol. 2005;15:175-84.

2. Malý J, Widimský J, Dulícek P. Venous thromboembolism prophylaxis in internal medicine. Vnitr Lek. 2009:55:190-5.

3. Yao JS. Varicose veins. Asian J Surg 2003;26:59-61

4. Sadick NS. Advances in the treatment of varicose veins: Ambulatory phlebectomy, foam sclerotherapy, laser and radiofrequency Closure. Dermatol Clin. 2005;23:443-55, vi.

5. Licun W, Gongjia S. Treatment of hemangioma with an angiogenesis inhibitor pingyangmycin. Indian Pediatr. 2000;37:636-9.

6. Duncan IC, Van Der Nest L. Intralesional bleomycin injections for the palliation of epistaxis in hereditary hemorrhagic telangiectasia. AJNR Am J Neuroradiol. 2004;25:1144-6.

7. Heller R, Jaroszeski MJ, Reintgen DS, Puleo CA, DeConti RC, Gilbert RA, Glass LF. Treatment of cutaneous and subcutaneous tumors with electrochemotherapy using intralesional bleomycin. Cancer. 1998;83:148-57.

8. Alomrani AH, Maghraby GM, Alanazi FK, Mohanna MA, Alaiya AA, Alsarra IA. Liposomes for Enhanced Cytotoxic Activity of Bleomycin. Drug Development Res. 2011;72:265-73.
9. Blenkinsopp WK. Effect of injected sclerosant (tetradecyl sulphate of sodium) on rat veins. Angiologica. 1968;5:386-96.

10. Kanter A. Sequential injection of $3 \%$ sodium tetradecyl sulphate and $20 \%$ sodium chloride in the treatment of refractory varicosity of the greater saphenous vein. Dermatol Surg. 1995;21:355-6.

11. Christopher K, Gregoriadis G. Dehydration-Rehydration Vesicles: A Simple Method fo High Yield Drug Entrapment in Liposomes. Nature Biotechnology. 1984;2:979 - 84.

12. Tretbar LL. Injection sclerotherapy for spider telangiectasias: a 20-year experience with sodium tetradecyl sulfate. J Dermatol Surg Oncol. 1989;15:223-5.

13. Bergan JJ. Excision of varicose veins. lin: Ernst CB, Stanley JC, editors. Current therapy in vascular surgery. 4th ed. St. Louis: Mosby; 2001. p. 838-840.

14. Bergmeyer HU, Scheibe P, Wahlefeld AW. Optimization of methods for aspartate aminotransferase and alanine aminotransferase. Clin Chem. 1978;24:58-73.

15. Yagoda A, Mukherji B, Young C, Etcubanas E, Lamonte C, Smith JR, et al. Bleomycin, an antitumor antibiotic. Clinical experience in 274 patients. Ann Intern Med. 1972;77:861-70.

16. Chen Y, Chen J, Dong J, Jin Y. Comparing study of the effect of nanosized silicon dioxide and microsized silicon dioxide on fibrogenesis in rats. Toxicol Ind Health. 2004:20:21-7. 
17. Goldman M P, Bergan J J. Sclerotherapy: Treatment of Varicose and Telangiectatic Leg Veins. 3rd ed. St. Louis: Mosby; 2001. p. 1-6.

18. Bergan JJ, Pascarella L. Severe chronic venous insufficiency: Primary treatment with sclerofoam. Semin Vasc Surg. 2005;18:49-56.

19. Escribano JM, Juan J, Bofill R, Maeso J, Rodríguez-Mori A, Matas M. Durability of reflux-elimination by a minimal invasive CHIVA procedure on patients with varicose veins. A 3-year prospective case study. Eur J Vasc Endovasc Surg. 2003;25:159-63.

20. Brasic N, Lopresti D, McSwain H Endovenous laser ablation and sclerotherapy for treatment of varicose veins. Semin Cutan Med Surg. 2008;27:264-75.

21. Sarin S, Scurr JH, Coleridge Smith PD. Assessment of stripping of the long saphenous vein in the treatment of primary varicose veins. Br J Surg. 1992;79:889-93.

22. Dwerryhouse S, Davies B, Harradine K, Earnshaw JJ. Stripping the long saphenous vein reduces the rate of reoperation for recurrent varicose veins: five-year results of a randomized trial. J Vasc Surg. 1999;29:589-92.

23. Teruya TH, Ballard JL. New approaches for the treatment of varicose veins. Surg Clin North Am. 2004;84:1397-417, viii-ix.

24. Juhan C, Haupert S, Miltgen G,Barthelemy P, Eklof B. Recurrent varicose veins. Phlebology. 1990;5 201-11.

25. Jones L, Braithwaite BD, Selwyn D, Cooke S, Earnshaw JJ. Neovascularisation is the principal cause of varicose vein recurrence: results of a randomised trial of stripping the long saphenous vein. Eur J Vasc Endovasc Surg. 1996;12:442-5

26. Fegan WG. Continuous compression technique of injecting varicose veins. Lancet. 1963:2:109-12.

27. Lindsey B, Campbell WB. Rationing of treatment for varicose veins and use of new treatment methods: A Survey of practice in the United Kingdom. Eur J Vascular Endo Surg Extra. 2006;12:19-20.

28. Prasanthi D, Lakshmi PK. Vesicles - Mechanism of transdermal permeation: A Review. Asian J Pharm Clin Res 2012;5:18-25.

29. Chaudry G, Guevara CJ, Rialon KL, Kerr C, Mulliken JB, Greene AK, et al. Safety and Efficacy of Bleomycin Sclerotherapy for Microcystic Lymphatic Malformation. Cardiovasc Intervent Radiol. 2014;37:1476-81

30. Chan S, Middleton RK. Bleomycin treatment of warts. DICP. 1990;24:952-3.
MAILING ADDRESS:

Khalid M AlGhamdi

Dermatology Department,

Vitiligo Research Chair, College of Medicine,

King Saud University, P.O. Box 240997

11322 Riyadh, Saudi Arabia.

Email:kmgderm@gmail.com

How to cite this article: AlGhamdi KM, Kumar A, Ashour AE, AL-Rikabi AC, AlOmrani AH, Ahamed SS.Vascular sclerosing effects of bleomycin on cutaneous veins: a pharmacopathologic study on experimental animals. An Bras Dermatol. 2017;92(4):484-91. 